\title{
EXPERIMENTAL MURINE SCHISTOSOMIASIS MANSONI: ESTABLISHMENT OF THE CHRONIC PHASE OF THE DISEASE
}

\author{
RADOVAN BOROJEVIC
}

Departamento de Bioquímica, Instituto de Química, Universidade Federal do Rio de Janeiro, Caixa Postal 68021, 21944-970 Rio de Janeiro, RJ, Brasil

After the acute hyperergic phase of schistosomal infection, the chronic phase of the disease corresponds to the establishment of a relative equilibrium between the host and the parasite. This involves: (1) A shift from the predominance of the TH2 response observed in the acute phase, to the predominance of the THI response in the chronic phase of the disease, with modification of lymphokine and imunoglobulin secretions patterns. (2) Redistribution of hosts responses to parasite, with predominance of systemic controls in the acute phase, and a shift towards local tissue responses in the chronic phase. This redistribution relieves the hyperergic response involving the whole body of the host, and delimits cellular and molecular reactions to parasites to only those tissues that are directly involved by the adult parasites and their eggs. Mobilization of eosinophil granulocytes in schistosomal periovular granulomas is one of examples of this redistribution.

Key words: schistosomiasis mansoni - mice -- immune regulations

Schistosomiasis, as well as the large majority of parasitic infections, results in the establishment of a chronic disease: after the insidious and frequently unrecognized primary infection, the disease evolves into the longstanding chronic phase, where a relatively viable equilibrium is established between the host and the parasite. Even in regions hyperendemic for schistosomiasis, a major part of infected inhabitants have a subclinic or a mild hepatointestinal form of the disease. The establishment of this viable equilibrium involves a complex regulation of host responses to parasites. These regulations were the object of our studies, in the murine model of schistosomiasis.

The primary schistosomal infection passes through three distinct, relatively short phases, that correspond to the passage of schistosomules through the skin, the lungs, and their establishment in the mesenteric venous system. In all these phases, a number of immature worms die and disintegrate in the host's body, releasing antigenic substances, and causing mobilization of cells of the monomacrophagic lineage as well as the early lymphocyte response. However, only the beginning of oviposition, with the massive release of egg antigens, marks the full development of the acute phase of the disease.
Schistosome eggs, trapped in host tissues, induce formation of granulomas, that have been shown to be T-lymphocyte-mediated reactions of the delayed hypersensitivity type to the potent schistosome egg soluble antigens (Boros \& Warren, 1970). Mature schistosome worms do not replicate, but they are long-lived and the egg deposition is continuous along all the infection period. Despite this uninterrupted antigenic stimulation, a down regulation of the granulomatous response is observed in chronic schistosomiasis. It was originally described as "endogenous desensitization" (Domingo \& Warren, 1968). The size of granulomas decreases, in consequence of the down-regulation of lymphocyte and eosinophil mobilization (Moore et al., 1977). This is in agreement with the observed reduction of T-lymphocyte reactivity and secretion of major lymphokines (Colley, 1975). Transfer experiments have shown that this downregulation was mediated by $\mathrm{CD} 8+$ cells, and this experimental model has been consequently considered as a typical example of the T-mediated-T-suppression (Mathew \& Boros, 1986). This downregulation of granulomas is considered to be a hallmark of the establishment of the chronic phase of schistosomiasis.

Notwithstanding this downregulation of granulomatous response to eggs, several host re- 
actions to the parasite are enhanced in the chronic phase of the disease. Egg-antigens sequestration in granulomas is more efficient (Lichtenberg, 1964). This correlates well with the general increase of macrophagic functions: their differentiation into polykarions is accelerated (Borojevic et al., 1984), their proliferation in granulomas is enhanced, and they are induced to secrete monokines that were undetectable in the acute phase (Clark et al., 1988; Borojevic et al., 1989; El-Cheikh et al., 1991a). Simultaneously, the fibrotic reaction in granulomas is accelerated, with modification and increase of collagen deposition (Grimaud et al., 1987; Silva et al., 1989), indicating increased stimulation of connective tissue cells. The progressive splenomegaly with high levels of antibody secretion indicates also the increased B-cell help and induction of terminal differentiation of this cell line. These observations indicate that, besides the downregulation of T-cell functions, a complex modification of interactions among different cells involved in host reaction to parasite are to be observed along the evolution of the acute to the chronic phase of the disease.

Mossman \& Coffman (1989) have proposed an alternative model for modulation of immune responses in infectious diseases, based upon the equilibrium between the two subsets of T helper cells: TH1 and TH2 cells. This model has given a satisfactory explanation for "horizontal" spectra of immune responses and of morbidity, observed in inbred mouse strains in various infectious diseases, and for the fundamental differences between immune responses elicited by different infectious agents. Recent research on schistosomiasis have shown that the acute phase of schistosomiasis is largely dominated by the activation of TH2 lymphocyte subsets. Activation of TH2 cells is dependent upon oviposition and $\mathrm{T}$-cell stimulation with soluble egg antigens. A simultaneous active downregulation of TH1 subsets is observed (Pearce et al., 1991). This is consistent with high eosinophilia and high levels of IgE, dependent upon IL-5 and IL-4 respectively, that are characteristic of the acute schistosomal infection.

Considering the decrease of IL-2 secretion in periovular granulomas in the chronic phase of the disease, Stavitsky (1987) has tempted to adapt the model of two T-helper subsets to the modulation of immune response in schistosomiasis japonica. He has postulated that, during the schistosomal infection, functions of TH1 subset are progressively diminished whereas those of TH2 subset are maintained or increased. Although this hypothesis may explain the IL-2 decrease, it is not consistent with decrease of both eosinophilia and IgE levels in chronic schistosomiasis.

There are at present no cell markers available to distinguish in tissues $\mathrm{TH} 1$ and $\mathrm{TH} 2$ subsets, and their equilibrium may be only estimated indirectly, through determination of their specific secretory activity. This approach is hempered by the difficulty to monitor specific lymphokine activity in biological fluids, where we measure not only the sum of the secretory activities of various cell populations, but also the simultaneous clearing efficiency or inactivation of a specific lymphokine activity as e.g. the inactivation of IL- 2 by shedding of IL-2 receptor molecules from activated lymphocytes. On the other hand, each TH subset differentially and effectively increases the frequency of immunoglobulin isotype switching, so that the ratio between isotypes may be indicative of the predominance of one or the other subset. Quantification of the immunoglobulin isotypes present in sera is submitted to the same restrictions as lymphokine quantification. Alternatively, B-cells, effectively engaged in secretion of each immunoglobulin isotype, may be quantified in tissues. This information may give quite an accurate idea of the frequency in local Ig-isotype switch.

In recent research (El-Cheikh et al., 1991b), we have monitored proliferation (cell size estimation, detection of cellular DNA content, thymidine incorporation) and antibody isotype secretion (protein-A isotype-specific plaque forming cell assay-PFC) for lymphocyte populations determined by immunofluorescence and flow cytometry. We have observed a sustained blastogenic response of both B- and T-cells. Similar to polyclonal and non-specific responses in several parasitic diseases, a sustained and elevated production of IgM was observed throughout the infection: both in spleen and granulomas, the IgM specific PFC were by far the most numerous ones. The ratio among IgM secreting cells and the sum of all other $\mathrm{Ig}$ isotype secreting cells was remarkably constant: it varied from 6.0 to 6.7 in spleens of mice with acute, chronic and late chronic phases of the disease, and from 5.8 to 7.5 in hepatic periovular granulomas. 
The follow-up of isotype specific PFC in spleens of schistosome-infected mice along the evolution of the disease has shown divergent patterns for IgM and IgG2a that increased, and IgG1 and IgE that decreased. Consequently, the ratio $\operatorname{IgG} 2 \mathrm{a} / \mathrm{IgG} 1$ increased from 3.7 in normal mice, and 5.9 in mice with acute schistosomal infection, to 21.8 and 11.2 in its chronic and late chronic phases, respectively. A high $\mathrm{IgG} 2 \mathrm{a} / \mathrm{IgG} 1$ ratio is characteristic of immune reactions where the $\mathrm{TH} 1$ subsets predominate, as viral infections (Coutelier et al., 1987). We may conclude that the evolution of the acute to the chronic phase of schistosomiasis corresponds to the shift from the TH2 predominance in acute to the $\mathrm{TH} l$ predominance in the chronic phase. This is also consistent with enhanced macrophage functions that are in general associated with $\mathrm{TH} 1$ activation.

The increase of $\mathrm{CD} 8+$ cells, both in the spleen and in granulomas, observed in chronic schistosomiasis, is also similar to viral infections (El-Cheikh et al., 1991b). CD8+ cells have a lymphokine secretion pattern similar to TH1 cells. Since $T H 1$ and $T H 2$ cells can manifest a mutual downregulation, the ability of the transferred CD8+ cells to induce a chronic-like granulomatous reaction in mice with acute infection may be consistent with the proposed model.

Pearce et al. (1991) and Grzych et al. (1991) have shown that the $\mathrm{TH} 2$ response in acute schistosomiasis is consequent to lymphocyte stimulation by egg antigens, while the TH1 response is dependent upon stimulation with schistosomule or worm antigens. This is consistent with the generally accepted notion that the type of antigens and the pattern of their presentation to the immune system determine the dominance of TH subsets. However, the shift from $\mathrm{TH} 2$ to $\mathrm{TH} 1$ dominance in chronic schistosomiasis is not controlled by these mechanisms, since schistosome eggs are continuously deposited from the very beginning of the acute phase of the disease. In this context, the effect of increased numbers of CD8+ cells, observed in chronic schistosomiasis, upon the TH1/TH2 equilibrium, remains to be established.

In parallel to the shift from the $\mathrm{TH} 2$ to the TH1 dominance in chronic schistosomiasis, we have disclosed the simultaneous complementary phenomenon of redistribution of host's reactions to parasites. The acute hyperergic phase of infection is essentially systemic. It involves whole the body, with high circulating cytokines levels and hyperergic reaction in the bone marrow and spleen. The bone marrow eosinophilia reaches very high levels: more than $50 \%$ of the medullar cell population may be involved in production of eosinophils. All the eosinophils are readily mobilized towards the tissues involved by parasites, and only immature eosinophils may be observed in the blood circulation at this stage (El-Cheikh \& Borojevic, 1990). It has been shown that this eosinophilia is controlled by the IL5 , since the neutralization in vivo by monoclonal antibodies abolishes completely the eosinophil response to the helminth (Coffman et al., 1989).

We have shown that in chronic, but not in acute schistosomiasis, extramedullar eosinophilopoiesis may be observed in tissue inflammatory infiltrates and in granulomas (El-Cheikh et al., 1991a). This peripheral amplification of eosinophils is mediated by a cytokine, different from IL-5, IL-3 or GM-CSF, the secretion of which is induced in macrophages in the chronic phase of the disease. At this period, the medullar production of eosinophils is supplemented or taken over by local, peripheral proliferation, involving only those tissue where the host interacts with the parasite, and where the control of cell proliferation and activation acts in the paracrine manner. Consequently, the bone marrow is relieved and medullar eosinophilia drops to less than $10 \%$. Presence of mature eosinophils in blood circulation shows that the equilibrium is established between the production and mobilization of eosinophils into tissues. This viable equilibrium may be maintained for long periods of infection.

Similar redistribution may be observed for other cell lines. We have monitored in vitro myeloid precursors (GM-CFU) from bone marrow, spleen and granulomas, by soft-agar cultures stimulated with GM-CSF. While mycloid precursor numbers were fairly constant in the bone marrow, their number in the spleen and granulomas increased constantly from the acute to the chronic phase of the disease. Finally, we have observed a considerable plasmacytogenesis in the mesenterium of chronically-infected mice, producing locally antibody-secreting cells into the abdominal cavity. 
Schistosomiasis is essentially an abdominal disease, with direct involvement of mesenteric and portal venous systems and adjacent tissues. While the acute phase involves whole the body, the establishment of the chronic phase of the disease corresponds to an attempt of the host to delimit the reaction only to sites inhabited by parasites or involved by their eggs. Downregulation of systemic responses, induction of local macrophagic activity, and the passage from systemic to paracrine controls, act in the same sense: they result in the establishment of a viable equilibrium, characteristic of the chronic phase of this disease.

\section{REFERENCES}

BOROJEVIC, R.; NICOLA, M. H. \& SANTOS-DASILVA, C., 1984. Modulation of macrophage and polymorphonuclear granulocyte inflammatory reaction in experimental murine schistosomiasis mansoni. Cell. Mol. Biol., 30: 37-42.

BOROJEVIC, R.; PINTO, C. G.; EL-CHEIKH, M. C. \& DUTRA, H. S., 1989. Experimental murine schistosomiasis mansoni: hyperplasia of the monomacrophagic cell lineage and stimulation of myeloid proliferation by peripheral macrophages. Braz. J. Med. Biol. Res., 22: 579-586.

BOROS, D. L. \& WARREN, K. S., 1970. Delayed hypersensitivity-type granuloma formation and dermal reaction induced and elicited by a soluble factor isolated from Schistosoma mansoni eggs. J. Exp. Med., 132: 488-507.

CLARK, C. R. CHEN, B. D. M. \& BOROS, D. L., 1988. Macrophage progenitor cell and colonystimulating factor production during granulomatous schistosomiasis mansoni in mice. Infect. Immun., 56: $2680-2685$.

COFFMAN, R. L.; SEYMOUR, B. W. P.; HUDAK, S.; JACKSON, J. \& RENNICK, D., 1989. Antibody to interleukin-5 inhibits helminth-induced eosinophilia in mice. Science, 245: 308-311.

COLLEY, D. G., 1975. Immune response to a soluble egg antigen preparation during chronic primary infection with Schistosoma mansoni. J. Immunol., /15: 150-156.

COUTELIER, J. P.; VAN DER LOGT, J. T. M.; HEESSEN, F. A.; WARNIER, G. \& VAN SNICK, J., 1987. IgG2a restriction of murine antibodies elicited by viral infections. J. Exp. Med., 165: 64-69.

DOMINGO, E. O. \& WARREN, K. S., 1968. Endogenous desensitization: changing host granulomatous response to schistosome eggs at different stages of infection with Schistosoma mansoni. Am. J. Pathol,
52: 369-377.

EL-CHEIKH, M. C. \& BOROJEVIC, R., 1990. Extramedullar proliferation of eosinophil granulocytes in chronic schistosomiasis mansoni is mediated by a factor secreted by inflammatory macrophages. Infect. Immun., 58: 816-821.

EL-CHEIKH, M. C.; DUTRA, H. S. \& BOROJEVIC, R., 1991 a. Eosinophil granulocyte proliferation and differentiation in schistosomal granulomas are controlled by two cytokines. Lab. Invest., 64: 93-97.

EL-CHEIKH, M. C.; MINOPRIO, P. M.; DUTRA, H. S. \& BOROJEVIC, R, 1991b. Experimental murine schistosomiasis mansoni: lymphocyte subsets activity and establishment of the chronic phase of the disease. (submitted).

GRIMAUD, J. A.; BOROS, D. L.; TAKIYA, C.; MATHEW, R. C. \& EMONARD, H., 1987. Collagen isotypes, laminin, and fibronectin in granulomas of the livers and intestines of Schistosoma mansoni-infected mice. Am. J. Trop. Med. Hyg., 37: 335-344.

GRZYCH, J. M.; PEARCE, E.; CHEEVER, A.; CAULADA, Z. A.; CASPAR, S.; HEINY, S.; LEWIS, F. \& SHER, A., 1991. Egg deposition is the major stimulus for the production of $\mathrm{Th} 2$ cytokines in murine schistosomiasis mansoni. $J$. Immunol., 146: 1322-1327.

LICHTENBERG, F. VON, 1964. Studies on granuloma formation, antigen sequestration and destruction in the schistosome pseudotubercle. Am. J. Pathol., 45: 75-93.

MATHEW, R. C. \& BOROS, D. L., 1986. Anti-L3T4 antibody treatment suppresses hepatic granuloma formation and abrogates antigen-induced interleukin-2 production in Schistosoma mansoni infection. Infect. Immun., 54: 820-826.

MOORE, D. L.; GROVE, D. I. \& WARREN, K S., 1977. The Schistosoma mansoni egg granuloma: quantitation of cell populations. J. Pathol, 121: $41-50$.

MOSSMAN, T. R. \& COFFMAN, R. L., 1989. TH1 and TH2 cells: different patterns of lymphokine secretion lead to different functional properties. Annu. Rev. Immunol., 7: 145-173.

PEARCE, E. J.; CASPAR, P.; GRZYCH, J. M.; LEWIS, F. A. \& SHER, A., 1991. Downregulation of TH1 cytokine production accompanies induction of TH2 responses by a parasitic helminth, Schistosoma mansoni. J. Exp. Med., 173: 159-166.

SILVA, L. C.; MOURÃO, P. A. S. \& BOROJEVIC, R. 1989. Patterns of sulfated glycosaminoglycan synthesis and accumulation in hepatic granulomas induced by schistosomal infection. Exp. Mol. Pathol., 50: $411-420$.

STAVITSKY, A. B., 1987. Immune regulation in schistosomiasis japonica. Immunol. Today, 8: 228-233. 